\title{
PERFIL NUTRICIONAL DE CRIANÇAS PORTADORAS DO TRANSTORNO DO ESPECTRO AUTISTA
}

\section{Nutritional profile of children bearing autism spectrum disorder \\ Perfil nutricional de niños portadores de trastorno del espectro autista}

\author{
Maria Vanuza Caetano \\ Instituto Federal de Educação, Ciência e Tecnologia do Ceará - IFCE - Limoeiro do Norte (CE) - Brasil \\ Daniel Cordeiro Gurgel \\ Instituto Federal de Educação, Ciência e Tecnologia do Ceará - IFCE - Limoeiro do Norte (CE) - Brasil
}

\section{RESUMO}

Objetivo: Avaliar o estado nutricional e o consumo alimentar de crianças portadoras do transtorno do espectro autista (TEA). Métodos: O estudo teve abordagem de natureza quantitativa, descritiva, exploratória e transversal. Participaram 26 crianças, de 3 a 10 anos de idade, com diagnóstico do TEA, de ambos os sexos, atendidas no município de Limoeiro do Norte, Ceará. Os dados foram coletados através de entrevistas, ordenadas por um questionário sociodemográfico (idade, renda familiar, escolaridade dos participantes, tratamento psicofarmacológico, idade recebida do diagnóstico do TEA, classificação da CID-10 e histórico clínico); histórico nutricional; aplicação de 3 recordatórios de 24 horas; e medidas antropométricas (peso, altura, circunferência do braço e as dobras cutâneas tricipital e subescapular), com posterior cálculo do índice de massa corporal (IMC). Utilizou-se análise descritiva e as variáveis contínuas foram expressas em média \pm desvio padrão e coeficiente de variação. Resultados: Das crianças avaliadas, 10 (38,5\%) apresentaram sobrepeso $(23,1 \%, n=6)$ e obesidade $(15,38 \%, n=4)$ pelo IMC/I (Índice de Massa Corporal para Idade), bem como 10 crianças (38,5\%) apresentaram risco de sobrepeso. O consumo de energia (EER) esteve acima do recomendado para $14(53,85 \%)$ dos autistas. Identificou-se inadequação no consumo de vitamina $\mathrm{A}(77 \%$, $\mathrm{n}=20)$, vitamina $\mathrm{B}_{6}(58 \%, \mathrm{n}=15)$ e cálcio $(50 \%, \mathrm{n}=13)$. Conclusão: As crianças com o TEA demonstram elevados índices de sobrepeso, obesidade e elevada inadequação na ingestão de vitaminas e minerais.

Descritores: Avaliação Nutricional; Consumo de Alimentos; Transtorno Autístico.

\section{ABSTRACT}

Objective: To evaluate the nutritional status and dietary intake of children bearing the autism spectrum disorder (ASD). Methods: The study had a quantitative, descriptive, exploratory and cross-sectional approach. Twenty-six children aged 3 to 10 years, with diagnosis of ASD, of both sexes, attended to in the city of Limoeiro do Norte, Ceará State, Brazil, participated in the study. Data was collected through interviews, guided by a socioeconomic questionnaire (age, family income, participants'level of education, psychopharmacological treatment, age at diagnosis of ASD, ICD-10 coding, and clinical history); dietary history, application of three 24-hour recall, and anthropometric measurements (body weight, height, arm circumference and tricipital and subscapular skinfolds), with a subsequent calculation of the body mass index (BMI). Descriptive analysis was used and continuous variables were expressed as mean \pm standard deviation and coefficient of variation. Results: Of the children evaluated, $10(38.5 \%)$ were overweight $(23.1 \%, n=6)$ or obese $(15.38 \%, n=4)$ according to the BMI-forage, and a further 10 children (38.5\%) were at risk of overweight. The estimated energy requirement (EER) was above that recommended in $14(53.85 \%)$ of the autistic patients. The intake of vitamins $A(77 \%, n=20)$ and $B 6(58 \%, n=15)$ and calcium intake $(50 \%$, $n=13)$ were inadequate. Conclusion: Children with ASD evidence high overweight and obesity rates, and high inadequacy in vitamins and minerals intake.

Descriptors: Nutrition Assessment; Food Consumption; Autistic Disorder. 


\section{RESUMEN}

Objetivo: Evaluar el estado nutricional y el consumo de alimentos de niños portadores de trastorno del espectro autista (TEA). Métodos: Estudio de abordaje cuantitativo, descriptivo, exploratorio y transversal. Participaron 26 niños entre 3 y 10 años de edad, de ambos los sexos y con el diagnóstico de TEA que eran asistidos en el municipio de Limoeiro de Norte, Ceará. Se recogieron los datos a través de entrevistas ordenadas por un cuestionario sociodemográfico (edad, renta familiar, escolaridad de los participantes, tratamiento psicofarmacológico, edad que recibió el diagnóstico de TEA, clasificación de la CID-10 e historia clínica); el histórico nutricional; la aplicación de 3 recordatorios de 24 horas; y las medidas antropométricas (el peso, la altura, la circunferencia del brazo y los pliegues cutáneos tricipital y subescapular) con posterior cálculo del índice de masa corporal (IMC). Se utilizó el análisis descriptivo y las variables continuas se expresaron en media \pm desviación típica y coeficiente de variación. Resultados: De entre los niños evaluados, $10(38,5 \%)$ presentaron sobrepeso $(23,1 \%$, $n=6)$ $y$ obesidad $(15,38 \%, n=4)$ a partir del IMC/E (Índice de Masa Corporal para la edad), así como 10 niños (38,5\%) presentaron riesgo de sobrepeso. El consumo de energía (EER) estuvo por encima del recomendado para $14(53,85 \%)$ de los autistas. Se identificó como inadecuado el consumo de vitamina A $(77 \%, n=20)$, vitamina $B_{6}(58 \%, n=15)$ y calcio $(50 \%, n=13)$. Conclusión: Los niños con TEA han demostrado elevados índices de sobrepeso y obesidad e inadecuada ingesta de vitaminas y minerales.

Descriptores: Evaluación Nutricional; Consumo de Alimentos; Trastorno Autístico.

\section{INTRODUÇÃO}

O Transtorno do Espectro Autista (TEA) é um transtorno do desenvolvimento caracterizado por alterações na capacidade cognitiva e nas interações sociais que pode levar ainda a uma seletividade alimentar. Essa desordem apresenta diversidade de manifestações clínicas de alta complexidade, as quais podem estar relacionadas com inúmeras interações entre os genes, fatores epigenéticos e a exposição aos fatores ambientais ${ }^{(1)}$. A etiologia do TEA ainda é desconhecida, apesar de haver inúmeras hipóteses e já terem se passado mais de 70 anos desde os primeiros estudos e publicações sobre o tema ${ }^{(2)}$.

Na década de 1980-1990, a prevalência estimada era de 4-5/10.000 habitantes, aumentando para 30-60/10.000 na década 1990-2000. Segundo o Centers for Disease Control and Prevention (CDC), em 2015, houve uma prevalência do transtorno de 14,7 por 1000 ( 1 em 45) crianças com idade de 8 anos, afetando 1 a cada 42 meninos e 1 a cada 189 meninas ${ }^{(3)}$.

De acordo com o censo 2010 do Instituto Brasileiro de Geografia e Estatítica (IBGE) ${ }^{(4)}$, estima-se que haja 454.706 crianças com transtorno do espectro autista (TEA) no Brasil, com uma taxa de prevalência de uma para 150, na proporção de 3 homens para 1 mulher. No Ceará, os dados são imprecisos.

Diante desse cenário, os diferentes níveis de atenção poderiam delinear estratégias de promoção da saúde para os portadores de TEA e seus familiares, com o intuito de proporcionar atenção integral à saúde e promoção da qualidade de vida. Para tanto, é necessária a participação dos profissionais de saúde e dos gestores de políticas públicas ofertando cuidados básicos de saúde quanto ao diagnóstico, à prevenção de agravos e às ofertas de reabilitação e cuidados contínuos ${ }^{(5)}$.

As principais intervenções de promoção da saúde apontadas pela literatura, relacionadas aos autistas, consideram aspectos importantes de seu contexto (motoras, cognitivas, comunicação, expressão, socialização, psíquicas e nutricionais), visando à prevenção do agravo da deficiência e ao favorecimento de competências sociais para sua autonomia e independência, que visam melhorar suas vidas em geral $^{(6)}$.

Além das características mais marcantes percebidas nos portadores do transtorno do espectro autista (TEA), relacionadas, principalmente, ao falho desenvolvimento da linguagem e interação social, ainda há uma série de desordens gastrointestinais que podem acometer os autistas, como diminuída produção de enzimas digestivas, inflamações da parede intestinal e permeabilidade intestinal alterada, e todos esses fatores agravam os sintomas dos portadores da doença ${ }^{(7)}$.

A alimentação inadequada e a falta de equilíbrio energético são motivos de especial preocupação, pois a ingestão de micronutriente está estreitamente relacionada à ingestão de energia, sendo provável que as crianças cujo consumo de energia seja menor também sofram de deficiências de vitaminas e minerais ${ }^{(8)}$. Dados sugerem que as crianças autistas possuem de duas a três vezes mais chances de serem obesas do que adolescentes na população em geral ${ }^{9)}$. Portanto, a atividade física e os cuidados nutricionais são elementos valiosos na prevenção de doenças, como a obesidade infantil, para manutenção da independência funcional, participação social e qualidade de vida. O inadequado estado nutricional, a limitada variedade de alimentos e a gravidade da sintomatologia associada ao TEA podem causar significativo impacto na qualidade de vida dos pacientes, pais e cuidadores $^{(10)}$.

Desse modo, percebe-se uma relação ainda inconclusiva entre o TEA e os aspectos nutricionais, como o estado nutricional e comportamento alimentar. Portanto, pesquisas que aprimorem essa relação podem colaborar diretamente para a construção de evidências de qualidade e, consequentemente, fornecer adequadas estratégias de intervenções para pacientes e familiares. Neste contexto, o objetivo do trabalho foi avaliar o estado nutricional e o consumo alimentar de crianças portadoras do transtorno do espectro autista (TEA). 


\section{MÉTODOS}

Trata-se de um estudo de natureza quantitativa, descritiva, exploratória e transversal, realizado na associação de pais denominada Diamante Azul, do município de Limoeiro do Norte, Ceará, Brasil, no período de março a junho de 2017.

Incluíram-se na pesquisa crianças com idade entre 3 e 10 anos, de ambos os sexos, com diagnóstico do TEA de acordo com a classificação de doenças CID-10(11), segundo Manual de Diagnóstico e Estatística de Doenças Mentais da Academia Americana de Psiquiatria (DSM-IV-TR) $)^{(12)}$, e cujo pai ou responsável tenha permitido a participação através da assinatura do termo de consentimento livre e esclarecido.

Os dados foram coletados através de visita domiciliar, durante os meses de março a junho de 2017, na qual se realizou entrevistas, ordenadas por um questionário sociodemográfico composto por questões objetivas e subjetivas, histórico nutricional, três recordatórios de 24 horas (dois dias referente à semana e outro do final de semana), bem como, houve coleta das medidas antropométricas.

Realizou-se a entrevista após assinatura do Termo do Assentimento do Menor e Termo de Consentimento Livre e PósEsclarecido pelos pais das crianças participantes da pesquisa, sendo acentuados os aspectos pertinentes à autonomia, ao sigilo e à confidencialidade dos dados.

O questionário sociodemográfico foi aplicado a fim de identificar idade, renda familiar, escolaridade, tratamento psicofarmacológico, idade recebida do diagnóstico do TEA, classificação da CID-10 ${ }^{(11)} \mathrm{e} \mathrm{histórico} \mathrm{clínico} \mathrm{dos} \mathrm{pacientes.}$

Avaliou-se a estimativa do consumo de alimentos por meio do inquérito recordatório alimentar de 24 horas de três dias, todos aplicados de forma presencial na residência do participante. Este método consiste na obtenção de informações verbais, referente à ingestão alimentar das últimas 24 horas, contendo dados sobre os alimentos e bebidas, bem como peso/tamanho das porções consumidas ${ }^{(13)}$. Cabe ressaltar que o inquérito aplicado foi respondido pelos responsáveis das crianças. Determinouse o valor energético total (VET), bem como o cálculo das quantidades de carboidratos, proteína e lipídios, obedecendo a recomendação de $45 \%$ a $65 \%, 10 \%$ a $30 \%$, e $25 \%$ a $35 \%$, respectivamente ${ }^{(14)}$. Neste estudo, considerou-se margem de ingestão de $10 \%$ do recomendado, ou seja, foi considerada adequada a ingestão entre $90 \%$ a $110 \%$ do recomendado; inferior a $90 \%$, ingestão abaixo do recomendado; e, superior a 110\%, ingestão acima do recomendado.

Utilizou-se a Tabela Brasileira de Composição de Alimentos ${ }^{(15)}$ como referência para obtenção da composição nutricional dos alimentos. Após os cálculos, compararam-se os valores com as ingestões dietética de referência (DRIs) de acordo com a necessidade média estimada (EAR), ou Ingestão Adequada (AI), e Limite Superior Tolerável de Ingestão (UL) ${ }^{(16)}$, considerando sexo e idade de cada paciente. Avaliaram-se os seguintes micronutrientes: cálcio $(\mathrm{Ca})$, magnésio $(\mathrm{Mg})$, fósforo $(\mathrm{P})$, ferro $(\mathrm{Fe})$, potássio $(\mathrm{K})$, sódio $(\mathrm{Na})$, vitamina A (A), vitamina B1 (B1), vitamina B2 (B2), niacina, Vitamina B6 (B6), vitamina C (C) e ainda fibras e colesterol.

Os parâmetros antropométricos utilizados foram peso, altura, circunferência do braço e as dobras cutâneas tricipital e subescapular. Aferiu-se o peso em uma balança eletrônica do tipo plataforma digital (Toledo ${ }^{\circledR}$ ), com capacidade de $200 \mathrm{~kg}$. A estatura e a circunferência do braço (CB) foram aferidas utilizando-se fita métrica inextensível (Cescorf $\left.{ }^{\circledR}\right)$ com precisão de $1 \mathrm{~mm}$. Para aferição da estatura, os participantes eram encostados na parede, descalços com os pés paralelos, os tornozelos juntos, em posição ereta, os braços ao longo do corpo, com a cabeça posicionada de forma que a parte inferior da órbita ocular estivesse no mesmo plano do orifício externo do ouvido ${ }^{(17)}$. A circunferência braquial foi realizada no ponto médio entre o acrômio e o olecrano ${ }^{(18)}$. Para a coleta das dobras cutâneas (tricipital e subescapular), utilizou-se um adipômetro clínico (Cescorf®) que exerce pressão de $10 \mathrm{~g} / \mathrm{mm}^{2}$ com precisão de $1 \mathrm{~mm}$.

A partir desses parâmetros, realizou-se, então, a classificação do estado nutricional de cada participante, seguindo os índices peso para idade (P/I), estatura para idade (E/I), peso para estatura (P/E) e índice de massa corpórea para idade (IMC/I) descritos pelo SISVAM $^{(19)}$, segundo o escore Z. Para a medida da circunferência braquial, foram calculados os percentuais de adequação para: circunferência braquial $(\% \mathrm{CB})$, circunferência muscular do braço (\% CMB), circunferência muscular do braço corrigida $(\% \mathrm{CMBc})$ e dobra cutânea tricipital $(\% \mathrm{DCT})^{(20)}$. Para classificar a reserva de adiposidade, utilizaram-se os percentis de DCT (dobra cutânea tricipital), DCS (dobra Cutânea Sub- escapular) e SDTS (soma das dobras cutâneas tricipital e subescapular) ${ }^{(18)}$.

Utilizou-se o software Statistical Package for Social Sciences, versão 21.0 (SPSS® Inc, Chicago, IL), para análise descritiva, e as variáveis contínuas foram expressas em média \pm desvio padrão e coeficiente de variação.

O estudo recebeu aprovação do Comitê de Ética em Pesquisa (CEP) do Instituto Federal de Educação, Ciência e Tecnologia do Ceará (IFCE), conforme Parecer n 2.160.713.

\section{RESULTADOS}

Os participantes da pesquisa ( $\mathrm{n}=26)$, apresentaram idade média de 7 anos $( \pm 2)$, não estando nenhum participante com idade inferior aos três anos. O presente estudo identificou que 24 (92,31\%) crianças da amostra eram do sexo masculino e apenas $2(7,69 \%)$ do sexo feminino. Todas as crianças participantes da pesquisa possuíam diagnóstico fechado com classificação do CID-10 ${ }^{(11)}$. Destas, $24(92,4 \%)$ possuíam classificação para F84.0 (Autismo infantil) e $2(7,6 \%)$ se classificaram dentro do 
F84.0 mais associações F71.0 (Retardo mental moderado) e F90.0 (Transtornos hipercinéticos). Os dados demonstraram que $50 \%(\mathrm{n}=13)$ das famílias possuem renda familiar entre 1 a 1,5 salários mínimos.

Os resultados apontaram que $69,2 \%$ dos participantes $(n=18)$ faziam tratamento psicofarmacológico. Dentre os fármacos mais consumidos está a risperidona, com 42,3\% $(\mathrm{n}=11)$. Além do tratamento psicofarmacológico, $21(80,8 \%)$ crianças realizam terapias multiprofissionais com psicólogos, terapeutas ocupacionais e fonoaudiólogos, pelo menos uma vez por semana, como também toda a amostra frequenta escolas ou creches. (Tabela I).

Tabela I - Descrição dos dados socioeconômicos das crianças de 3 a 10 anos com diagnóstico de transtorno do espectro autista. Limoeiro do Norte, Ceará, 2017.

\begin{tabular}{|c|c|c|c|}
\hline Variáveis & Categorias & $\mathbf{n}$ & $\%$ \\
\hline \multicolumn{4}{|l|}{ Sexo } \\
\hline & Feminino & 2 & 7,69 \\
\hline & Masculino & 24 & 92,31 \\
\hline \multicolumn{4}{|l|}{ Idade } \\
\hline & De 3 a 4 anos & 4 & $15,4 \%$ \\
\hline & De 5 a 6 anos & 8 & $30,8 \%$ \\
\hline & De 7 a 8 anos & 8 & $30,8 \%$ \\
\hline & De 9 a 10 anos & 6 & $23,1 \%$ \\
\hline \multicolumn{4}{|c|}{ Renda Familiar } \\
\hline & $<1$ salário mínimo & 1 & $3,8 \%$ \\
\hline & 1 salário mínimo & 13 & $50,0 \%$ \\
\hline & De 1,5 até 2 salários mínimos & 8 & $30,8 \%$ \\
\hline & Mais de 2 salários mínimos & 4 & $15,4 \%$ \\
\hline \multicolumn{4}{|c|}{ Possui Laudo Diagnóstico } \\
\hline & Sim & 26 & $100,0 \%$ \\
\hline & Não & 0 & $0,0 \%$ \\
\hline \multicolumn{4}{|c|}{ Idade de Diagnóstico } \\
\hline & $<3$ anos & 5 & $19,2 \%$ \\
\hline & De 3 a 4 anos & 16 & $61,5 \%$ \\
\hline & De 5 a 6 anos & 2 & $7,7 \%$ \\
\hline & De 7 a 8 anos & 2 & $7,7 \%$ \\
\hline & De 9 a 10 anos & 1 & $3,8 \%$ \\
\hline \multicolumn{4}{|c|}{ Classificação no CID-10 } \\
\hline & F84.0 & 24 & $92,4 \%$ \\
\hline & F84.0/F71.0 & 1 & $3,8 \%$ \\
\hline & F84.0/F90.0 & 1 & $3,8 \%$ \\
\hline \multicolumn{4}{|c|}{ Uso de Medicação } \\
\hline & Sim & 18 & $69,2 \%$ \\
\hline & Não & 8 & $30,8 \%$ \\
\hline \multicolumn{4}{|c|}{ Medicação utilizada } \\
\hline & Rispiridona & 11 & $42,3 \%$ \\
\hline & Ritalina & 4 & $15,4 \%$ \\
\hline & Neuleptiil & 3 & $11,5 \%$ \\
\hline & Depaquer & 3 & $11,5 \%$ \\
\hline & Outros & 5 & $19,2 \%$ \\
\hline \multicolumn{4}{|c|}{ Frequenta escola } \\
\hline & Sim & 26 & $100 \%$ \\
\hline & Não & 0 & $0 \%$ \\
\hline \multicolumn{4}{|c|}{ Terapia multiprofissional } \\
\hline & Sim & 21 & $80,8 \%$ \\
\hline & Não & 5 & $19,2 \%$ \\
\hline
\end{tabular}

Legenda: F84.0: Autismo infantil; F71.0: Retardo mental moderado; F90.0: Transtornos hipercinéticos

Na avaliação do consumo de energia estabeleceram-se faixas de classificação. Os valores inferiores a 90\% da recomendação foram considerados consumo abaixo da recomendação; de $90 \%$ a $110 \%$, adequado; e acima da recomendação para $110 \%$. Os valores encontrados podem ser observados na Figura 1. 


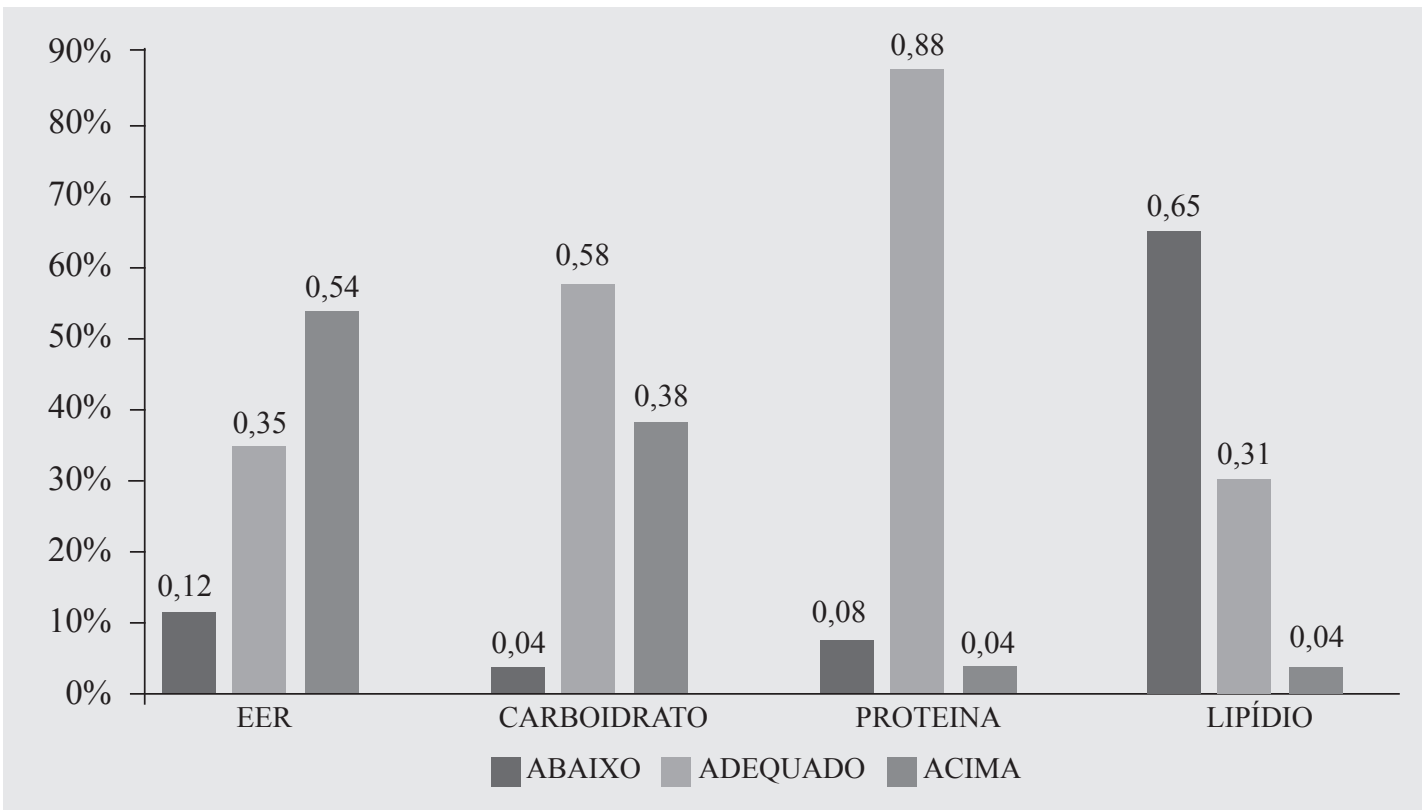

Figura 1 - Descrição entre o percentual de adequação do gasto calórico (EER) e do consumo de macronutrientes em crianças autistas. Limoeiro do Norte, Ceará, 2017.

Legenda: EER: Necessidade energética

Para avaliação da adequação dos macronutrientes, utilizou-se a recomendação da $\mathrm{IOM}^{(14)}$, que indica: $45 \%$ a $65 \%$ para carboidrato, $10 \%$ a $30 \%$ para proteína, e $25 \%$ a $35 \%$ para lipídios, com faixa etária 4 a 18 anos.

Os resultados para carboidrato $(57,69 \%, \mathrm{n}=15)$ e proteínas $(88,46 \%, \mathrm{n}=23)$ se encontravam adequados, o que difere para o valor para lipídios $(65 \%, \mathrm{n}=17)$, que estava abaixo do recomendado. Entretanto, os resultados encontrados para o consumo de energia (EER) estão acima do recomendado para $14(53,85 \%)$ dos participantes da pesquisa.

A seguir, a Tabela II demonstra a adequação de minerais e vitaminas, respectivamente, encontrados com base nas recomendações das DRIs ${ }^{(16)}$.

Tabela II - Descrição dos resultados do consumo de minerais e vitaminas em crianças autistas segundo as DRIs (ingestões dietética de referência). Limoeiro do Norte, Ceará, 2017.

\begin{tabular}{|c|c|c|c|c|c|c|c|c|c|c|c|c|}
\hline \multirow[b]{2}{*}{ Consumo } & \multicolumn{6}{|c|}{ Minerais } & \multicolumn{6}{|c|}{ Vitaminas } \\
\hline & $\begin{array}{c}\mathrm{Ca} \\
(\mathrm{mg})\end{array}$ & $\begin{array}{l}\text { Mg } \\
(\mathrm{mg})\end{array}$ & $\begin{array}{c}P \\
(\mathrm{mg})\end{array}$ & $\begin{array}{c}\mathrm{Fe} \\
(\mathrm{mg})\end{array}$ & $\begin{array}{c}\mathrm{K} \\
(\mathrm{mg})\end{array}$ & $\begin{array}{l}\mathrm{Na} \\
(\mathrm{mg})\end{array}$ & $\begin{array}{c}\text { A } \\
(\mathrm{mcg})\end{array}$ & $\begin{array}{c}\text { B1 } \\
(\mathrm{mg})\end{array}$ & $\begin{array}{c}\text { B2 } \\
(\mathrm{mg})\end{array}$ & $\begin{array}{l}\text { Niac. } \\
\text { (mg) }\end{array}$ & $\begin{array}{c}\text { B6 } \\
\text { (mg) }\end{array}$ & $\begin{array}{c}\mathrm{C} \\
(\mathrm{mg})\end{array}$ \\
\hline POS ADE & $\begin{array}{l}46,15 \% \\
(n=12)\end{array}$ & $\begin{array}{c}0,00 \% \\
(\mathrm{n}=0)\end{array}$ & $\begin{array}{l}80,77 \% \\
(n=21)\end{array}$ & $\begin{array}{c}88,46 \% \\
(n=23)\end{array}$ & $\begin{array}{c}100,00 \% \\
(n=26)\end{array}$ & $\begin{array}{c}26,92 \% \\
(n=7)\end{array}$ & $\begin{array}{c}23,08 \% \\
(n=6)\end{array}$ & $\begin{array}{l}84,62 \% \\
(n=22)\end{array}$ & $\begin{array}{c}69,23 \% \\
(n=18)\end{array}$ & $\begin{array}{c}26,92 \% \\
(n=7)\end{array}$ & $\begin{array}{c}42,31 \% \\
(n=11)\end{array}$ & $\begin{array}{c}69,23 \% \\
(n=18)\end{array}$ \\
\hline $\begin{array}{l}\text { POS } \\
\text { INAD }\end{array}$ & $\begin{array}{l}50,00 \% \\
(n=13)\end{array}$ & $\begin{array}{c}42,31 \% \\
(n=11)\end{array}$ & $\begin{array}{c}19,23 \% \\
(n=5)\end{array}$ & $\begin{array}{c}7,69 \% \\
(n=2)\end{array}$ & $\begin{array}{c}0,00 \% \\
(\mathrm{n}=0)\end{array}$ & $\begin{array}{c}69,23 \% \\
(n=18)\end{array}$ & $\begin{array}{l}76,92 \% \\
(n=20)\end{array}$ & $\begin{array}{c}15,38 \% \\
(n=4)\end{array}$ & $\begin{array}{c}30,77 \% \\
(n=8)\end{array}$ & $\begin{array}{c}19,23 \% \\
(n=5)\end{array}$ & $\begin{array}{l}57,69 \% \\
(n=15)\end{array}$ & $\begin{array}{c}30,77 \% \\
(n=8)\end{array}$ \\
\hline $\begin{array}{l}\text { POS } \\
\text { NOC }\end{array}$ & $\begin{array}{c}3,85 \% \\
(\mathrm{n}=1)\end{array}$ & $\begin{array}{c}57,69 \% \\
(\mathrm{n}=15)\end{array}$ & $\begin{array}{c}0,00 \% \\
(\mathrm{n}=0)\end{array}$ & $\begin{array}{c}3,85 \% \\
(n=1)\end{array}$ & $\begin{array}{l}0,00 \% \\
(\mathrm{n}=0)\end{array}$ & $\begin{array}{c}3,85 \% \\
(n=1)\end{array}$ & $\begin{array}{c}0,00 \% \\
(\mathrm{n}=0)\end{array}$ & $\begin{array}{c}0,00 \% \\
(\mathrm{n}=0)\end{array}$ & $\begin{array}{c}0,00 \% \\
(n=0)\end{array}$ & $\begin{array}{c}53,85 \% \\
(n=14)\end{array}$ & $\begin{array}{c}0,00 \% \\
(\mathrm{n}=0)\end{array}$ & $\begin{array}{c}0,00 \% \\
(\mathrm{n}=0)\end{array}$ \\
\hline
\end{tabular}

Legenda: POS ADE: possivelmente adequado; POS INAD: possivelmente inadequado; POS NOC: possivelmente nocivo; Ca: cálcio; Mg: magnésio; P: fósforo; Fe: ferro; K: potássio; Na: sódio; mg: miligrama; vitamina A; B1: vitamina B1; B2: vitamina B2; Niac.: niacina; B6: vitamina B6; C; vitamina C; mcg: micrograma

Entre os minerais, identificou-se uma possível inadequação no consumo de cálcio $(50 \%, n=13)$ e sódio $(69,23 \%, n=18)$. O magnésio obteve resultado possivelmente nocivo $(57,69 \%, \mathrm{n}=15)$. Já para o fósforo, ferro e potássio, encontraram-se resultados possivelmente adequados, com $80,77 \%(n=21), 88,46 \%(n=23)$ e $100 \%(n=26)$ respectivamente. 
Os dados apresentados para o consumo de vitaminas demostraram uma inadequação para vitamina $\mathrm{A}(77 \%, \mathrm{n}=20)$ e $\mathrm{B} 6$ $(58 \%, \mathrm{n}=15)$, estando abaixo das recomendações.

Foi encontrado o consumo dentro do recomendado para as vitaminas B1(84,62\%, n=22), B2 (69,23\%, $\mathrm{n}=18)$ e C (69,23\%, $\mathrm{n}=18$ ), estando apenas a niacina como possivelmente nociva.

Os resultados encontrados para a porcentagem de fibras consumidas pelas crianças foram 10 (38\%) adequadas, entretanto 9 (35\%) foram nocivas e 7 (27\%) inadequadas. O consumo de fibra dietética pode estar associado aos hábitos intestinais dos participantes à medida que $20(77 \%)$ realizavam a defecação pelo menos uma vez todos os dias, enquadrando-se como 2 ou 3 na Escala de Bristol.

A Tabela III demonstra o estado nutricional dos portadores de TEA e destaca que 10 (38,5\%) apresentaram sobrepeso $(23,1 \%, n=6)$ e obesidade $(15,38 \%, n=4)$ pelo IMC/I (Índice de Massa Corporal para Idade), outras 10 crianças $(38,5 \%)$ apresentaram risco de sobrepeso e 20 crianças $(76,98 \%)$ risco de obesidade. Resultado semelhante aos dados referentes ao parâmetro de IMC/I, o índice peso para estatura $(\mathrm{P} / \mathrm{E})$, no qual a soma do percentual de crianças com risco de sobrepeso $(15,38 \%, n=4)$, sobrepeso $(26,9 \%, n=7)$ e obesidade $(19,23 \%, n=5)$ ultrapassa o percentual de crianças com diagnóstico de eutrofia $(38,46 \%, \mathrm{n}=10)$.

$\mathrm{O}$ índice $\mathrm{P} / \mathrm{I}$ evidenciou que a maioria das crianças $(57,69 \%, \mathrm{n}=15)$ estava com peso adequado para idade, não deixando de ser relevantes os valores referentes às crianças que apresentaram peso elevado para idade $(38,5 \%, n=10)$. A E/I apresentou-se como adequada em toda a amostra avaliada.

Tabela III - Classificação do estado nutricional de crianças autistas. Limoeiro do Norte, Ceará, 2017.

\begin{tabular}{|c|c|c|c|c|}
\hline \multicolumn{5}{|c|}{ Classificação do estado nutricional } \\
\hline \multicolumn{5}{|c|}{ IMC para idade (IMC/I) } \\
\hline Magreza & Eutrofia & Risco de sobrepeso & Sobrepeso & Obesidade \\
\hline $3,85 \%(n=1)$ & $19,23 \%(n=5)$ & $38,46 \%(n=10)$ & $23,08 \%(n=6)$ & $15,38 \%(n=4)$ \\
\hline \multicolumn{5}{|c|}{ Peso para idade $(\mathrm{P} / \mathrm{I})$} \\
\hline & $\begin{array}{c}\text { Baixo peso para idade } \\
3,8 \%(n=1)\end{array}$ & $\begin{array}{l}\text { Peso adequado para idade } \\
57,69 \%(n=15)\end{array}$ & $\begin{array}{l}\text { Peso elevado para idade } \\
38,51 \% 9(\mathrm{n}=10)\end{array}$ & \\
\hline \multicolumn{5}{|c|}{ Peso para estatura $(\mathrm{P} / \mathrm{E})$} \\
\hline & Eutrofia & Risco de sobrepeso & Sobrepeso & Obesidade \\
\hline & $38,46 \%(n=10)$ & $15,38 \%(n=4)$ & $26,93 \%(n=7)$ & $19,23 \%(n=5)$ \\
\hline \multicolumn{5}{|c|}{ Estatura para idade (E/I) } \\
\hline \multicolumn{5}{|c|}{$\begin{array}{l}\text { Estatura adequada para idade } \\
\qquad 100 \%(\mathrm{n}=26)\end{array}$} \\
\hline
\end{tabular}

Legenda: n: número de crianças; IMC: Índice de Massa Corporal

O perfil nutricional do público avaliado, segundo os percentuais de $\% \mathrm{CB}, \% \mathrm{CMB}, \% \mathrm{CMBc}, \% \mathrm{DCT}$, DCT, DCS e SDTS está representado na Tabela IV. A classificação nutricional através do $\% \mathrm{CB}$, indica que $38,46 \%(\mathrm{n}=10)$ apresentaram classificação eutrófica. Entretanto, não pode ser considerado um valor positivo se este for associado aos 42,31\% ( $\mathrm{n}=11)$ referentes a sobrepeso e obesidade, bem como 19,23\% ( $\mathrm{n}=5)$ de desnutridos moderados e leves. Para reserva de gordura, de acordo com as DCT, DCS e SDCTS, encontrou-se que o maior número dos pacientes apresenta percentual de gordura em excesso (obesidade), 65,38\% $(\mathrm{n}=17), 76,92 \%(\mathrm{n}=20)$ e $76,92 \%(\mathrm{n}=20)$, respectivamente. 
Estes resultados colaboram para a importância da avaliação nutricional (antropométrica, da composição corporal) dentro da rotina clínica de pacientes com TEA e seus familiares, sempre considerando as características singulares de cada paciente.

Tabela IV - Classificação da composição corporal de crianças autistas. Limoeiro do Norte, Ceará, 2017.

\begin{tabular}{|c|c|c|c|c|}
\hline \multicolumn{5}{|c|}{ Classificação de composição corporal } \\
\hline \multicolumn{5}{|c|}{ Circunferência braquial (\%CB) } \\
\hline Desnutrição moderada & Desnutrição leve & Eutrofia & Sobrepeso & Obesidade \\
\hline $7,69 \%(\mathrm{n}=2)$ & $11,54 \%(n=3)$ & $38,46 \%(n=10)$ & $19,23 \%(\mathrm{n}=5)$ & $23,08 \%(\mathrm{n}=6)$ \\
\hline \multicolumn{5}{|c|}{ Circunferência muscular do bracco (\%CMB) } \\
\hline Desnutrição grave & \multirow{2}{*}{\multicolumn{2}{|c|}{$\begin{array}{l}\text { Desnutrição moderada } \\
7,69 \%(\mathrm{n}=2)\end{array}$}} & Desnutrição leve & Eutrofia \\
\hline $11,54 \%(\mathrm{n}=3)$ & & & $26,92 \%(\mathrm{n}=7)$ & $53,85 \%(n=14)$ \\
\hline \multicolumn{5}{|c|}{ Circunferência muscular do braço corrigida (\%CMBc) } \\
\hline & Normal & & Desnutrição leve moderada & \\
\hline & $73,08 \%(n=19)$ & & $26,92 \%(n=7)$ & \\
\hline \multicolumn{5}{|c|}{ Dobra cutânea tricipital (\%PCT) } \\
\hline Desnutrição grave & Desnutrição leve & Eutrofia & Sobrepeso & Obesidade \\
\hline $7,69 \%(\mathrm{n}=2)$ & $3,85 \%(n=1)$ & $7,69 \%(n=2)$ & $7,69 \%(n=2)$ & $73,08 \%(\mathrm{n}=19)$ \\
\hline \multicolumn{5}{|c|}{ Dobra cutânea tricipital (DCT) } \\
\hline & Desnutrido & Eutrofia & Obesidade & \\
\hline & $3,85 \%(n=1)$ & $30,77 \%(n=8)$ & $65,38 \%(n=17)$ & \\
\hline \multicolumn{5}{|c|}{ Dobra cutânea subescapular (DCS) } \\
\hline & Eutrofia & & Obesidade & \\
\hline & $23,08 \%(n=6)$ & & $76,92 \%(n=20)$ & \\
\hline \multicolumn{5}{|c|}{ Soma das dobras cutâneas tricipital e subescapular (SDTS) } \\
\hline & Desnutrido & Eutrofia & Obesidade & \\
\hline & $3,85 \%(\mathrm{n}=1)$ & $19,23 \%(\mathrm{n}=5)$ & $76,92 \%(n=20)$ & \\
\hline
\end{tabular}

Legenda: n: número de crianças

\section{DISCUSSÃO}

No presente estudo, a amostra analisada $(\mathrm{n}=26)$ apresentou uma maior prevalência para o sexo masculino, corroborando os achados de estudos anteriores ${ }^{(13,21)}$, que relatam maior incidência em pesquisas epidemiológicas do autismo, com uma média de 3,5 a 4 meninos para cada 1 menina; como também destacam o autismo como sendo 4 vezes mais comum em garotos do que em garotas, numa escala de 5/10.000. A média de idade em que as crianças fecharam o diagnóstico foi aos 4 anos, sendo que nenhuma crianças apresentou idade menor que três anos no presente estudo, fato que se associa à dificuldade de confirmação do diagnóstico precoce desse transtorno ${ }^{(13)}$. O diagnóstico para o TEA é eminentemente clínico, e deve ser realizado a partir dos critérios da CID-10 $0^{(11)}$, através de anamnese com os pais, responsáveis legais e/ou cuidadores, bem como da observação clínica do comportamento(3).

Os dados avaliados na presente pesquisa mostram que $50 \%$ das famílias possuíam renda familiar entre 1 a 1,5 salários mínimos. Esse resultado é similar ao observado em outro estudo ${ }^{(22)}$, em que $56 \%$ das famílias entrevistadas tinham renda familiar semelhante.

Encontrou-se elevada prevalência de autistas $(69,2 \%)$ que fazem tratamento psicofarmacológico na atual pesquisa. Dentre os fármacos mais utilizados, está a risperidona, com $42,3 \%$, a qual tem evidenciado resultados positivos, que incluem a redução de agressividade, da irritabilidade e do isolamento; porém, seus efeitos adversos mais comuns são sonolência, tontura, sialorreia e ganho ponderal. O uso de risperidona está associado também a alterações metabólicas, como aumento da resistência à insulina, hiperglicemia, hipertensão arterial e dislipidemia ${ }^{(23)}$. Os fármacos de ação no sistema nervoso central não são utilizados com a finalidade de cura, mas de alívio de sintomas. A participação de $100 \%$ da amostra em escolas ou creches auxilia a criar alternativas para a inclusão social, pois o contato com terapias multiprofissionais amplia suas formas de se expressarem e se comunicarem $^{(24)}$.

Os resultados do presente estudo se encontravam adequados para carboidrato $(57,69 \%)$ e proteínas $(88,46 \%)$, o que difere para o valor para lipídios (65\%), que se encontrava abaixo do recomendado. Vale ressaltar que dietas com quantidades insuficientes de gordura podem levar a redução da absorção de alguns micronutrientes, como vitaminas lipossolúveis ${ }^{(25)}$. Entretanto, os resultados encontrados para o consumo de energia (EER) estavam acima do recomendado para 53,85\% dos autistas. Tais achados possivelmente se associam aos frequentes erros alimentares característicos do transtorno, como a seletividade e a compulsão alimentar. A seletividade alimentar varia de criança para criança, constituindo um problema quando interfere na rotina diária e social ${ }^{(26)}$. Relatos e testemunhos de pessoas com TEA sugerem que as características sensoriais dos alimentos, como o odor, a textura, a cor e a temperatura, possam contribuir para a seletividade alimentar ${ }^{(27,28) .}$ 
É muito comum as crianças autistas possuírem deficiências nutricionais, pois a maioria apresenta uma alimentação monótona. Porém, mesmo que a criança possua uma dieta variada e adequada nutricionalmente, ela precisa ser capaz de executar três funções básicas que, infelizmente, não são feitas pela maioria: digerir e quebrar adequadamente o alimento até uma forma absorvível, absorver os nutrientes através do TGI saudável e converter os nutrientes em uma forma utilizável em nível celular ${ }^{(25)}$.

As deficiências de micronutrientes mais comuns nos TEA são das vitaminas B1, B3, B5, B6, B9, B12, A e dos minerais cálcio $(\mathrm{Ca})$, zinco $(\mathrm{Zn})$, selênio $(\mathrm{Se})$ e magnésio $(\mathrm{Mg})^{(29)}$. No atual trabalho, pôde-se observar uma possível inadequação $(50 \%)$ de cálcio $(\mathrm{Ca})$, que está diretamente associado a diversas funções orgânicas, como modulação de sinais de transdução, metabolismo de produção de energia e proliferação celular, estando os sintomas resultantes de sua deficiência associados à: ansiedade, depressão, hiperatividade, agitação, alucinações, irritabilidade, nervosismo, agressão, estresse crônico, dificuldade de aprendizagem e perda de memória ${ }^{(30)}$. Em relação à ingestão de vitamina $\mathrm{A}$, o resultado da atual investigação apontou para uma possível inadequação. Essa deficiência, em crianças, pode causar falha de crescimento e danos oculares, como a xeroftalmia ${ }^{(30)}$.

Nutrientes como a vitamina B6 são de extrema importância para a metilação, transulfatação e sulfatação, que representam um conjunto de atividades bioquímicas que não funcionam adequadamente nos portadores com TEA. Quando da limitação dessas transformações metabólicas, os neurotransmissores não são adequadamente ativados, ocasionando sintomas de ansiedade, depressão, déficit de atenção e transtorno do sono. Isto associado ao maior consumo de alumínio, mercúrio, glutamato e várias substâncias artificiais ingeridas na alimentação, favorecem o acúmulo no organismo e proporcionam alterações cerebrais que acarretam irritabilidade, agressividade, hiperatividade ${ }^{(13)}$. A limitação do consumo desses nutrientes junto à conversão das enzimas parece ser uma das razões para a deficiência de componentes essenciais para o organismo das crianças autistas, como: sulfato, cisteína, taurina e glutationa, levando aos prejuízos da maioria dos processos metabólicos encontrados ${ }^{(25)}$.

A ingestão adequada de outras vitaminas, como B1 e niacina, é de grande importância para as crianças autistas, já que sua deficiência é caracterizada por sinais neurológios, podendo intensificar os sintomas relacionados ao transtorno, já que impede a conversão do acetaldeído (substância neurotóxica) nas crianças autistas, prejudicando sua eliminação pelo organismo. Isto pode afetar estruturas cerebrais, vindo a interferir no desenvolvimento neural dos autistas ${ }^{(23)}$.

Além da grande importância associada à avaliação dos micronutrientes, deve ser dada atenção ao papel desempenhado pelas fibras dietéticas na modulação da saúde intestinal. O consumo adequado de fibras deve proporcionar um funcionamento normal do intestino, prevenir câncer relacionado à dieta e diminuir a concentração sérica de colesterol para redução de risco de doença cardiovascular. Sua inadequação pode afetar as funções neurológicas por diversos mecanismos, refletindo em muitos dos sintomas vistos no TEA ${ }^{(25)}$. Estudos ${ }^{(25,31)}$ demonstraram que a digestão dos alimentos pelas crianças autistas está prejudicada, normalmente, devido à baixa sulfatação e transulfatação, processos essenciais na digestão, para a integridade da mucosa intestinal, a destoxificação e o equilíbrio da microbiota, podendo interferir na absorção de micronutrientes e ocasionar baixos níveis de minerais e vitaminas, entre outros compostos.

Além dos parâmetros relacionados à ingestão alimentar, a avaliação física e antropométrica adequadas podem contribuir para o melhor entendimento do quadro clínico dos autistas. Desse modo, com relação ao estado nutricional das crianças portadoras do TEA, trabalhos ${ }^{(13,32)}$ têm sido realizados para avaliar o perfil nutricional dessa população.

Observações clínicas mostram que essas crianças apresentam maior risco de excesso de peso, pois possuem grandes dificuldades em praticar exercícios físicos de forma estruturada, além do isolamento social, o que possibilita o aumento de sedentarismo e corrobora os dados desta pesquisa, em que todos os participantes não praticam nenhuma atividade física. Porém, o sobrepeso e a obesidade são problemas de saúde pública na população em geral, pois a incidência de muitas doenças crônicas na vida adulta está diretamente ligada à obesidade na infância ${ }^{(33)}$.

Na criança, a obesidade aumenta o risco de problemas em curto e longo prazos, como diabetes, doenças cardiovasculares e psicossociais. Estudo internacional indica que crianças e adolescentes com TEA podem ser particularmente vulneráveis a essas alterações ponderais ${ }^{(10)}$. Avaliou-se 30 crianças de uma escola especial de Campo Grande, Brasil e observou-se que quatro $(13,3 \%)$ estavam obesas e sete $(23,3 \%)$ com baixo peso. Como também avaliaram 23 crianças e adolescentes autistas e observaram que três $(13 \%)$ apresentavam baixo peso, cinco $(21,7 \%)$ tinham sobrepeso e seis $(26,1 \%)$ exibiam obesidade ${ }^{(7)}$. Embora os métodos de avaliação apresentados estabeleçam um perfil nutricional geral de pacientes portadores de TEA, faz-se necessária à associação de métodos de avaliação da composição corporal, pois permitem obter um diagnóstico nutricional mais preciso.

A adequação do percentual da CB é um parâmetro nutricional antropométrico recomendado pela OMS para estimativa da proteína musculoesquelética total, fornecendo índice de depósito de gordura e massa muscular local, e pode ser considerada uma medida independente, sendo uma das medidas mais utilizadas na avaliação nutricional ${ }^{(34)}$. O percentual de adequação da CMB avalia a reserva de tecido muscular sem correção da área óssea (não leva em consideração o diâmetro do osso). $\mathrm{O}$ diagnóstico encontrado para esse parâmetro aponta que 53,85\% das crianças com TEA do atual estudo estavam eutróficas. Em contrapartida, 46,15\% apresentavam desnutrição leve à desnutrição grave. A utilização dessa fórmula para o cálculo da 
adequação da CMB pode subestimar a perda muscular em até 20 a $25 \%$, uma vez que a área óssea é incluída no cálculo, mascarando assim a perda corporal.

O diagnóstico apresentado para o percentual de adequação da $\mathrm{CMBc}$, que avalia a reserva de tecido muscular corrigindo a área óssea (leva em consideração o diâmetro do osso), apresentou-se normal para 78,03\% $(\mathrm{n}=19)$ das crianças, refletindo mais adequadamente a verdadeira magnitude das mudanças do tecido muscular do que o percentual de adequação da $\mathrm{CMB}^{(20)}$. O estado nutricional, segundo o percentual de adequação da DCT, revela uma maior prevalência para obesidade com 73,08\% $(\mathrm{n}=19)$. Essa dobra cutânea é a mais utilizada na prática clínica para monitoramento do estado nutricional por ser mais representativa da distribuição de gordura corporal, independentemente da idade e do sexo, pois se correlaciona de forma significativa com o peso corporal e a massa gorda ${ }^{(18,20)}$. De acordo com a $\mathrm{OMS}^{(11)}$, tem-se notado aumento da prevalência de obesidade na infância e na adolescência, independentemente do método de classificação antropométrico utilizado.

Esse fato demanda atuações voltadas para a promoção da saúde, como uma política de alimentação saudável, ações que incentivem a atividade física regular e atividades culturais de lazer. Os estudos ainda não determinaram o tratamento ideal que engloba o contexto nutricional, controle comportamental, medicação, aspectos físicos e educacionais ${ }^{(29)}$. A intervenção dietética tem como objetivo melhorar a saúde física e bem-estar desses indivíduos, sendo essencial o acompanhamento nutricional junto às crianças autistas, contribuindo na correção de erros alimentares, bem como na promoção da saúde e da qualidade de vida. $\mathrm{O}$ diagnóstico precoce é o único consenso em todo o mundo no que diz respeito ao autismo ${ }^{(35)}$.

O desenvolvimento de novas pesquisas e a continuação de novos estudos é importante para melhorar a forma de abordagem profissional; consequentemente, também a qualidade de vida e a saúde desses pacientes. O presente estudo apresenta algumas limitações, pois a amostra é de uma área limitada do nordeste do país e pode não representar as mesmas características para outros grupos de crianças com TEA.

\section{CONCLUSÃO}

As crianças com o Transtorno do Espectro Autista avaliadas demonstraram elevados índices de sobrepeso e obesidade, repertório alimentar limitado, elevada inadequação na ingestão de vitaminas (A e B6) e do mineral cálcio, o que pode estar associado ao alto consumo de alimentos ricos em calorias e pobres em micronutrientes.

\section{CONFLITO DE INTERESSES}

Não houve conflito de interesses no presente estudo.

\section{REFERÊNCIAS}

1. Willsey AJ, State MW. Autism spectrum disorders: from genes to neurobiology. Curr Opin Neurobiol. 2015;30:92-9.

2. Tchaconas A, Adesman A. Autism spectrum disorders: a pediatric overview and update. Curr Opin Pediatr. 2013;25(1):13044.

3. Centers for Disease Control and Prevention. Prevalence of autism spectrum disorder among children aged 8 years - autism and developmental disabilities monitoring network, 11 sites, United States, 2010. MMWR Surveill Summ. 2014;63(2):121.

4. Instituto Brasileiro de Geografia e Estatítica. Censo demográfico 2010. Rio de Janeiro: IBGE; 2010.

5. Brasil. Constituição da República Federativa do Brasil de 1988. Brasília; 1988.

6. Cardellini DMC, Lacerda E, Zimmermann V. Movimento psicanálise, autismo e saúde pública. Boletim Online. 2013 [acesso em 2017 Dez 20]. Disponível em: http://www.sedes.org.br/Departamentos/Psicanalise/index.php?apg=b_visor\&p $\mathrm{ub}=24 \&$ ordem $=3$ \&origem $=$ abertas $\&$ itema $=2$.

7. Kummer A, Barbosa IG, Rodrigues DH, Rocha NP, Rafael MS, Pfeilsticker L,et al. Frequência de sobrepeso e obesidade em crianças e adolescentes com autismo e transtorno do déficit de atenção/hiperatividade. Rev Paul Pediatr. 2016;34(1): 71-7.

8. Domingues G. Relação entre medicamentos e ganho de peso em indivíduos portadores de autismo e outras síndromes relacionadas [dissertação]. Rio Grande do Sul: Universidade do Rio Grande do Sul; 2007.

9. Abreu LC. Condições relacionadas à obesidade secundária na interface do crescimento e desenvolvimento. Rev Bras Crescimento Desenvolv Hum. 2011;21(1):34-8. 
10. Zuchetto AT, Cavalcante TM, Pimenta RA, Zanon PA, Nasser JP. Avaliação da composição corporal de crianças e jovens com deficiência. Rev Port Ciênc Desporto. 2014;245-56.

11. Organização Mundial de Saúde. Classificação estatística internacional de doenças e problemas relacionados à saúde (Kortmann): CID-10. 10.ed. São Paulo; 2000. p.361-362.

12. American Psychiatric Association. American Psychiatric Association Diagnostic and Statistical Manual of Mental Disorders. $5^{\text {th }}$ ed. Arlington: American Psychiatric Publishing; 2013.

13. Grokoski KC. Composição corporal e avaliação do consumo e do comportamento alimentar em pacientes do transtorno do espectro autista [monografia]. Porto Alegre: Universidade de Porto Alegre; 2016.

14. Institute of Medicine of The National Academies (IOM). Committee on Food Marketing and the Diets of Children and Youth. Food Marketing to Children and Youth: Threat or Opportunity Whashington, 2006. p. 2-13.

15. Núcleo de Estudo e Pesquisa em Alimentos. Tabela Brasileira de Composição de Alimentos - TACO. $4^{\mathrm{a}}$ ed. rev. e ampl. Campinas: UNICAMP; 2011.

16. Institute of Medicine (USA). Dietary Reference Intakes (DRIs): recommended dietary allowances and adequate intakes, vitamins. Washington: National Academy of Sciences; 2004.

17. Jelliffe DB. Evaluación del estado de nutrición de la comunidad. Ginebra: Organización Mundial de la Salud; 1968.

18. Frisancho AR. Anthrometric standards for the assessment of growth and nutritional status. Ann Arbor: University of Michigam Press; 1990.

19. Ministério da Saúde (BR), Coordenação Geral da Política de Alimentação e Nutrição. Vigilância Alimentar e Nutricional: SISVAN. Orientações para a coleta e análise de dados antropométricos em serviços de saúde. Norma Técnica - SISVAN. Material Preliminar. Brasília: Ministério da Saúde; 2008.

20. Blackburn GL, Thornton PA. Nutritional assessment of the hospitalized patient. Med Clin North Am. 1979;63(5):1110315.

21. Kortmann GML. Aprendizagem da criança autista e suas relações familiares e sociais: Estratégias educativas [monografia]. Porto Alegre: Universidade Federal do Rio Grande do Sul; 2013.

22. Ministério da Saúde (BR), Secretaria de Atenção à Saúde, Departamento de Ações Programáticas Estratégicas. Diretrizes de Atenção à Reabilitação da Pessoa com Transtornos do Espectro do Autismo. Brasília: Ministério da Saúde; 2013.

23. Galling B, Correll CU. Do antipsychotics increase diabetes risk in children and adolescentes. Expert Opin Drug Saf. 2015;14(2):219-41.

24. Ministério da Saúde (BR), Secretaria de Ciência, Tecnologia e Insumos Estratégicos. CONITEC. Risperidona no Transtorno do Espectro do Autismo (TEA): Relatório de Recomendação da Comissão Nacional de Incorporação de Tecnologias no SUS -CONITEC -123. Brasília: Ministério da Saúde; 2014.

25. Reichow, B. Overview of meta-analyses on early intensive behavioral intervention for young children with Autism Spectrum Disorders. J Autism Dev Disord. 2012;42(4):512-20.

26. Huke V, Kent A, Morgan JF, Saeid IS, Turk J. autism spectrum disorders in eating disorder populations: a systematic review. Eur Eat Disord Rev. 2013;21(5):345-51.

27. Castro K, Faccioli LS, Baronio D, Gottfried C, Perry IS, Riesgo R. Body composition of patients with autism spectrum disorder through bioelectrical impedance. Nutr Hosp. 2017;34(4):875-9.

28. Curtin C, Jojic M, Bandini LG. obesity in children with autism spectrum disorders. Harv Rev Psychiatry. 2014;22(2):93103.

29. Oliveira ATD. Intervenção nutricional no Autismo [monografia]. Portugal: Universidade do Porto; 2012.

30. Vitolo MR. Nutrição da gestação ao envelhecimento. Rio de Janeiro: Editora Rubio; 2012.

31. Hyman SL, Stewart PA, Schmidt B, Cain U, Lemcke N, Foley JT. Nutrient intake from food in children with Autism. Pediatrics. 2012;130(Suppl 2):145-53.

32. Marí-Bauset S, Lopis-González A, Zazpe-Garcia I, Mari-Sanchis A, Morales-Suárez-Varela M. Nutritional status of children with autism spectrum disorders (ASDs): a case-control study. J Autism Dev Disord. 2015; 45(1):203-12. 
33. Meguid NA, Kandeel WA, Wakeel KE, El-Nofely AA. Anthropometric assessment of a Middle Eastern group of autistic children. World J Pediatr.2014;10(4):318-23.

34. Ogden CL, Carroll MD, Kit BK, Flegal KM. Prevalence of childhoodand adult obesity in the United States, $2011-2012$. JAMA. 2014;311(8):806-14.

35. Anagnostou E, Zwaigenbaum L, Szatmari P, Fombonne E, Fernandez B A, Woodbury-Smith M, et al. Autism spectrum disorder: advances in evidence-based practice. CMAJ. 2014;186(7):509-19.

\section{Endereço para correspondência:}

Maria Vanuza Caetano

Rua Estevão Remígio de Freitas, 1145

Bairro: Monsenhor Otávio

CEP: 62930-000 - Limoeiro do Norte - CE - Brasil

E-mail: vanuza.caetano@hotmail.com 